\title{
Metastatic sarcomatoid renal cell carcinoma to the mandible treated with Sorafenib
}

\author{
Kazuhiro Murakami*, Kazuhiko Yamamoto, Kumiko Aoki, Ikuyo Fukumoto, Tsutomu Sugiura, \\ Tadaaki Kirita
}

Department of Oral and Maxillofacial Surgery, Nara Medical University, Kashihara, Japan

Email: ${ }^{*}$ murakami@naramed-u.ac.jp

Received 10 May 2012; revised 18 June 2012; accepted 27 June 2012

\begin{abstract}
A case of metastatic sarcomatoid renal cell carcinoma to the mandible treated with Sorafenib is reported. A 76-year-old man consulted us for hyposthesia of the right lower lip. Panorama $\mathrm{X}$-ray film showed a radiolucent lesion in the right mandibular body. A diagnosis of metastatic tumor to the mandible from the left kidney was made after evaluation by computed tomography and positron emission tomography, which also revealed multiple bone metastases. After radiotherapy for mandibular and thoracic lesions, nephrectomy was performed. Histological diagnosis was sarcomatoid renal cell carcinoma. Interferon therapy was performed but was not effective; therefore, a molecular targeted drug, Sorafenib, was administered. Sorafenib effectively inhibited the growth of oral and other metastatic lesions for 10 months. Quality of life was relatively well maintained with tolerable adverse effects. The patient survived for as long as 2 years after appearance of the first symptom.
\end{abstract}

Keywords: Sarcomatoid Renal Cell Carcinoma; Oral Metastatic Lesion; Molecular Targeted Therapy; Sorafenib

\section{INTRODUCTION}

Metastatic tumors to the oral cavity are very uncommon and represent approximately $1 \%$ of oral neoplasms [1]. Renal carcinoma is the third most common infraclavicular neoplasm that metastasizes to the oral cavity, following that of lung and breast carcinoma [2]. Renal cell carcinoma that has metastasized at the time of initial diagnosis has an extremely poor prognosis, and more than $80 \%$ of patients die within 1 year [3]. Renal cell carcinoma with a sarcomatoid component often shows local invasion and distant metastasis. The prognosis is worse than that of other types of tumors [4,5] with a median survival time of 6.8 months [6].

${ }^{*}$ Corresponding author.
The introduction of molecular targeted therapies has revolutionized the treatment approach to metastatic renal cell carcinoma. The use of a molecular targeted drug, Sorafenib, was approved in April 2008 in Japan. Compared with cytokine-based immunotherapy, molecular targeted therapy shows higher response rates at primary and metastatic sites, and a favorable safety profile [7].

We reported a case of metastatic sarcomatoid renal cell carcinoma to the mandible, which was treated with Sorafenib in addition to nephrectomy, radiotherapy, and interferon therapy.

\section{CASE REPORT}

A 76-year-old man consulted a dental clinic with a complaint of hyposthesia of the right lower lip. He had noticed the hyposthesia 2 months previously; however, the symptom did not improve. He was referred to our hospital for a complete medical examination in September, 2008. He had a history of hypertension and had been using tobacco and alcohol for 50 years. His face was symmetric and the skin and oral mucosa in the right buccal region were normal (Figure 1). Swelling of submandibular lymph nodes was not observed. Hyposthesia

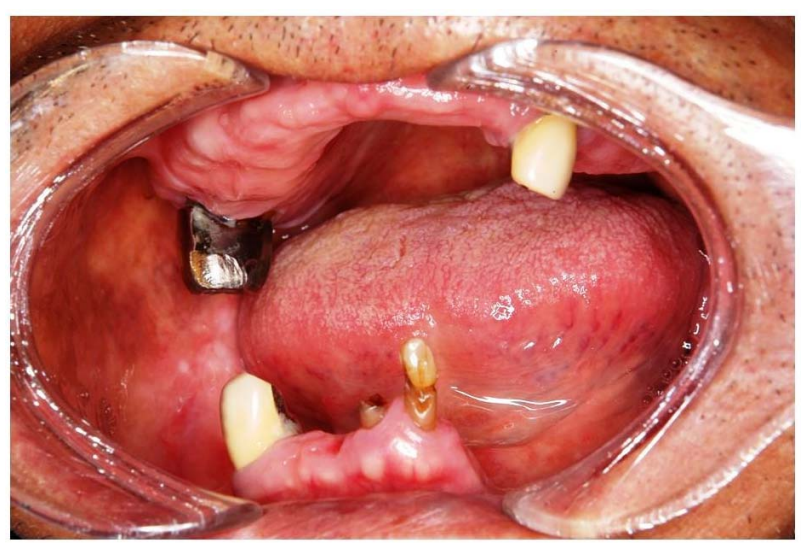

The right molar region of the mandible was covered with normal mucous membrane.

Figure 1. Oral finding at the first visit. 
was observed in the right mental region. Panorama X-ray film showed a diffuse radiolucent lesion of $30 \times 20 \mathrm{~mm}$ in the right mandibular body (Figure 2). Computed tomography (CT) demonstrated a $30 \mathrm{~mm}$ diffuse radiolucent lesion in the right mandibular body (Figure 3), and a $20 \mathrm{~mm}$ radiolucent lesion in the first thoracic vertebrae. On radioisotope examination (Tc99m), Tc99m was concentrated in the right mandibular body, the upper thoracic vertebrae, the right sacrum, the right 9th rib, and the left superior pole of the kidney. On positron emission tomography, $18 \mathrm{~F}-\mathrm{FDG}$ was concentrated in the right mandibular body (SUV max 12.2), the first thoracic vertebrae (standard uptake value (SUV) max 5.6), and the superior pole of the left kidney (SUV max 12.5) (Figure 4). Chest and abdominal CT showed a $78 \times 76 \mathrm{~mm}$ aneurysm in the abdominal aorta, multiple lymph nodes swelling at the bilateral hilus pulmonis and mediastinum,

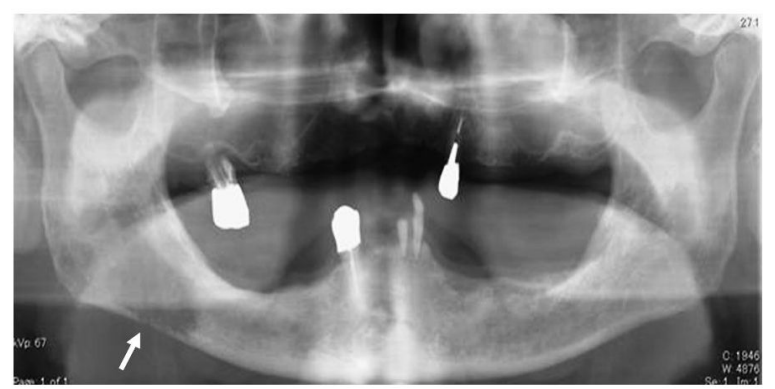

A diffuse radiolucent lesion of $30 \times 20 \mathrm{~mm}$ (arrow) was observed in the right mandibular body.

Figure 2. Panoramic X-ray finding at the first visit.

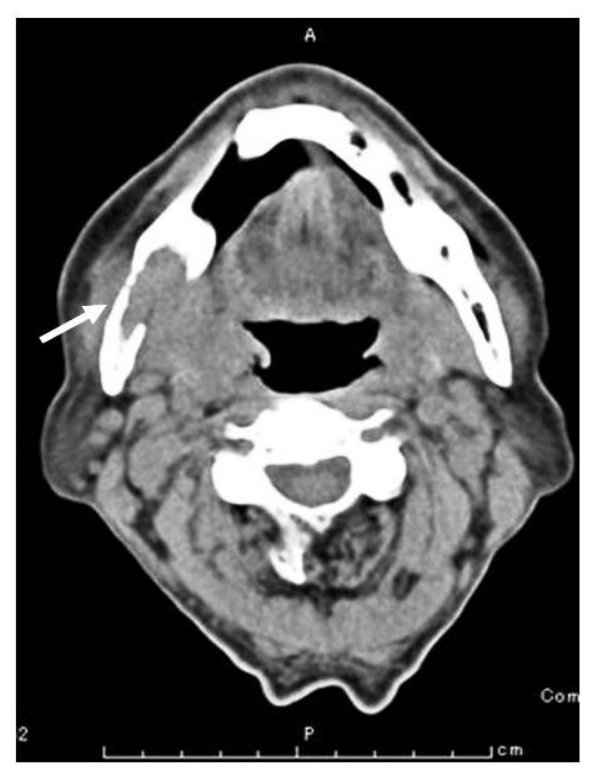

A $30 \mathrm{~mm}$ diffuse radiolucent lesion (arrow) in the right mandibular body with destruction of lingual cortical bone was evident.

Figure 3. Computed tomography finding of the mandible at the first visit. and a $75 \mathrm{~mm}$ heterogeneous mass at the superior pole of the left kidney (Figure 5). Blood chemistry findings were all within normal limits. These findings led to the clinical diagnosis of metastatic tumor of the mandible from the left kidney, although characteristic clinical symptoms of renal carcinoma such as abdominal pain, hematuria, and abdominal mass were not observed. The patient was referred to Cardiovascular Surgery, Urology and Radiology Departments for further evaluation.

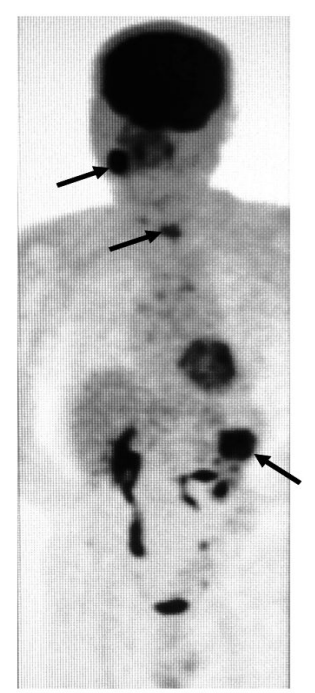

18 F-FDG was concentrated in the first thoracic vertebrae (standard uptake value (SUV) max 5.6), the right mandibular body ((SUV) $\max 12.2)$, and the left superior pole of the kidney ((SUV) max 12.5) (arrow).

Figure 4. Positron emission tomography findings at the first visit.

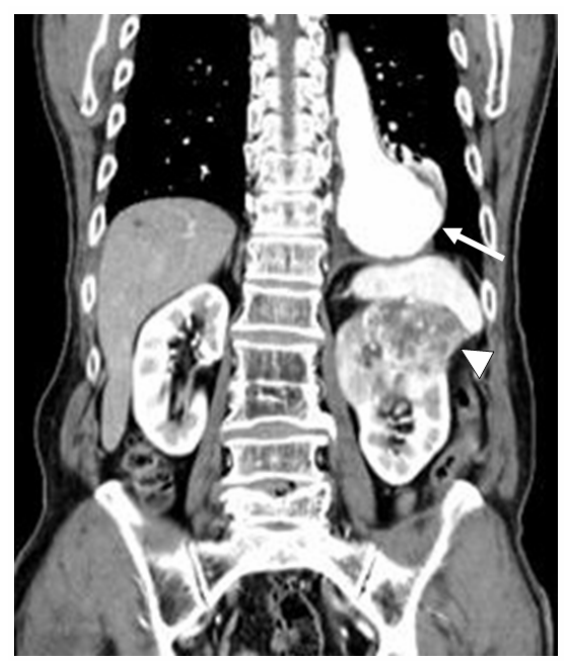

Chest and abdominal CT showed a $78 \times 76 \mathrm{~mm}$ aneurysm in the abdominal aorta (arrow) and a $75 \mathrm{~mm}$ diffuse radiolucent area at the left superior pole of the kidney (arrowhead).

Figure 5. Chest and abdominal coronal CT finding 
Endovascular aneurysm repair was performed by a stent graft for the abdominal aortic aneurysm before treatment for the renal carcinoma (Figure 6). The patient then underwent radiation therapy for the tumor of the mandible (total $37.5 \mathrm{~Gy}$ ) and the first thoracic vertebrae; however, the mandibular tumor enlarged, and pathological fracture of the mandible occurred. Fortunately, the fracture site was not mobile and oral intake was possible. After radiation, the patient underwent cytoreductive radical nephrectomy of the left kidney. Histopathologic diagnosis was sarcomatoid renal cell carcinoma, G3 pT3b (Figure 7). Interferon therapy (IFN- $\gamma 3 \times 10^{6}$ units/day) was carried out after nephrectomy, but showed no marked effect on bone metastatic lesions. Therefore, the patient was administered a molecular targeted drug, Sorafenib $(800 \mathrm{mg} /$ day $)$. Sorafenib inhibited the growth of all distant metastatic lesions for 10 months. The metastatic lesion in the mandible also remained dormant. Oral intake was not disturbed, although the mandible was gradually deviated toward the fracture side. The dose was reduced to $400 \mathrm{mg} /$ day because a hand-foot skin reaction developed 1.5 months after administering the agent (Figure 8).

Other metastatic lesions became evident at the right clavicle, the right upper arm, the left 9th rib, the right iliac bone, the lung field and the sacrum 10 months after the start of molecular targeted therapy. To control hypercalcemia due to osteolysis of multiple metastatic sites, Zoledronate was administered. The mandibular lesion also expanded markedly. Oral mucous membrane ruptured and a necrotic tumor were exposed in the oral cavity with occasional oozing (Figure 9); therefore, radiation therapy was performed to inhibit the tumor growth and to prevent possible massive hemorrhage (total 30 Gy). Sorafenib therapy was continued after a short cessation during radiation therapy. Severe adverse events from Sorafenib developed a month after radiation. Gastrointestinal perforation was managed with decompression through a gastric tube in combination with antibiotics, $\gamma$-globulin, and Omeprazole therapy. Hypothyroidism was controlled with Levothyroxine. To reduce these side effects, Sorafenib was replaced with Sunitinib; however, the patient died of cancerous cachexia 2 years after developing hyposthesia of the lower lip.

\section{DISCUSSION}

Metastatic neoplasms to the oral cavity occur more often in patients over 60 years old [8,9]. In approximately $20 \%$ of cases, these tumors were found before the primary tumors, and most commonly developed in the molar region of the mandible [9-14]. Good blood supply to the

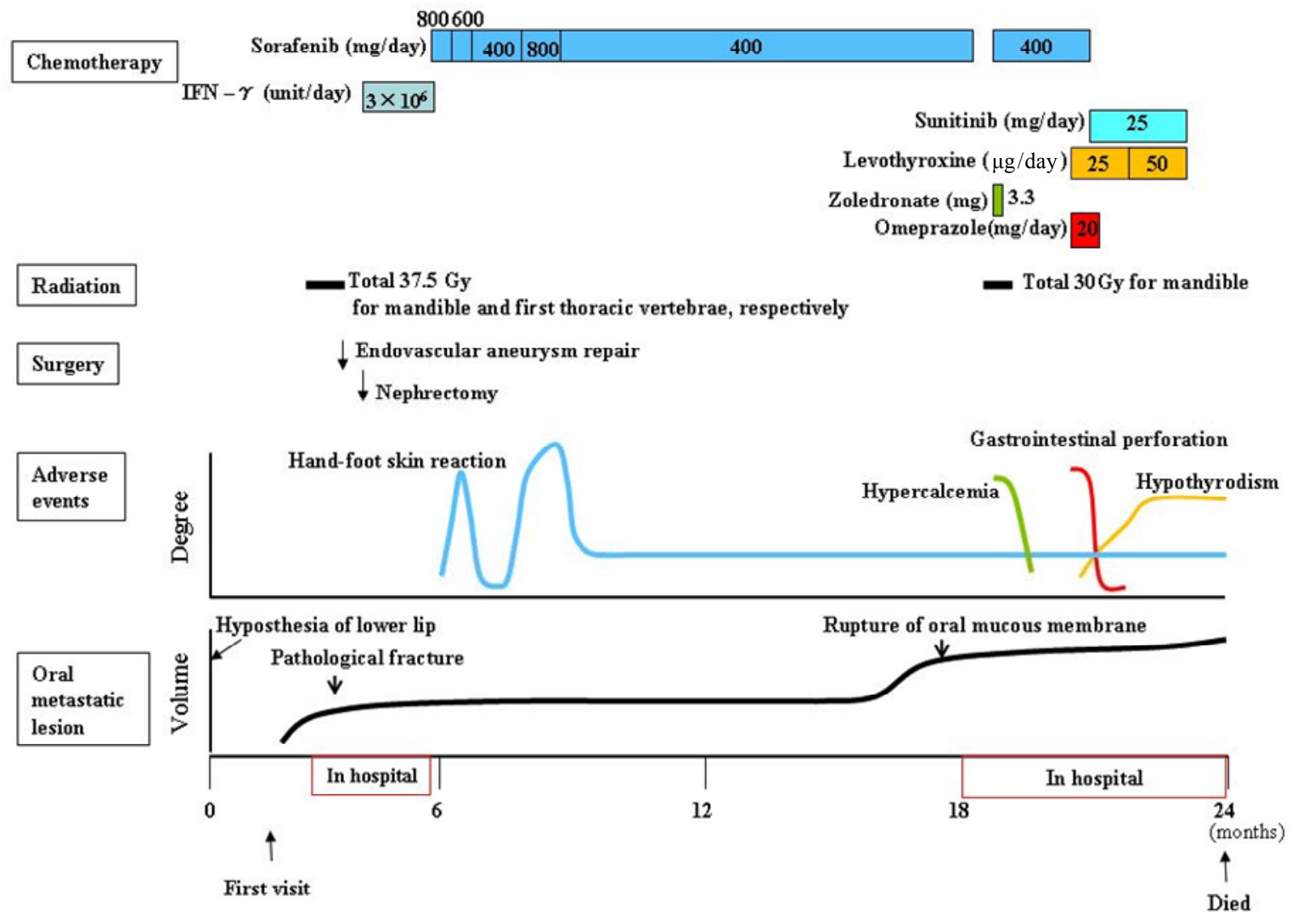

Figure 6. Clinical course. 


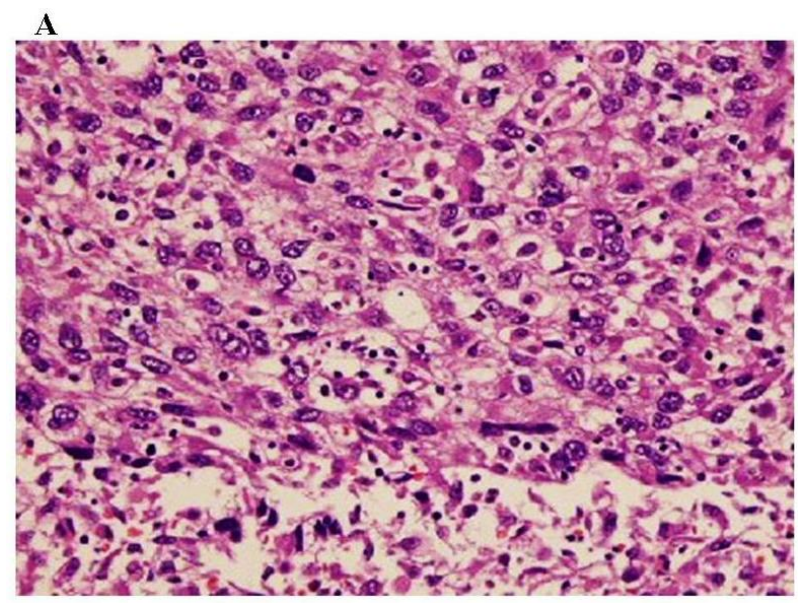

B

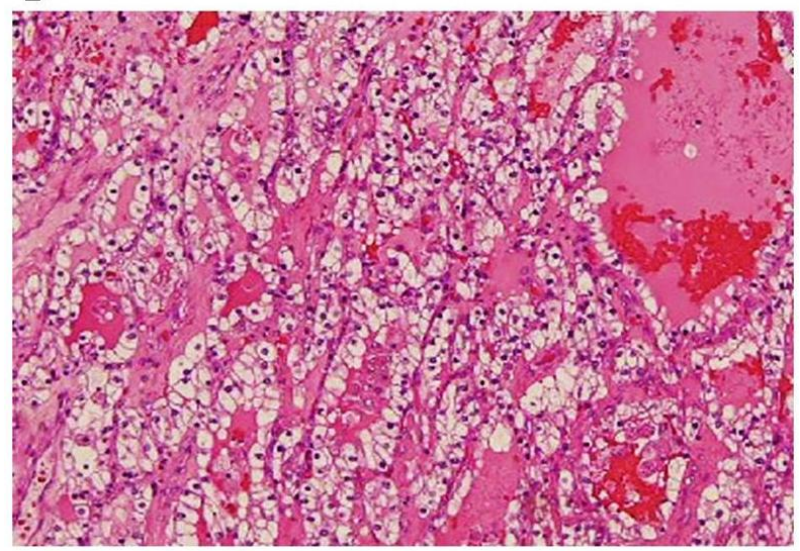

A: Sarcomatoid component: Pleomorphic and atypical cells proliferated accompanying necrosis in the sarcomatoid component, which occupied about $90 \%$ of the tumor (H \& E, $\times 200$ ); B: Clear cell component: Proliferation of clear tumor cells was partly observed(H \& E, ×200).

Figure 7. Histological findings of the tumor.

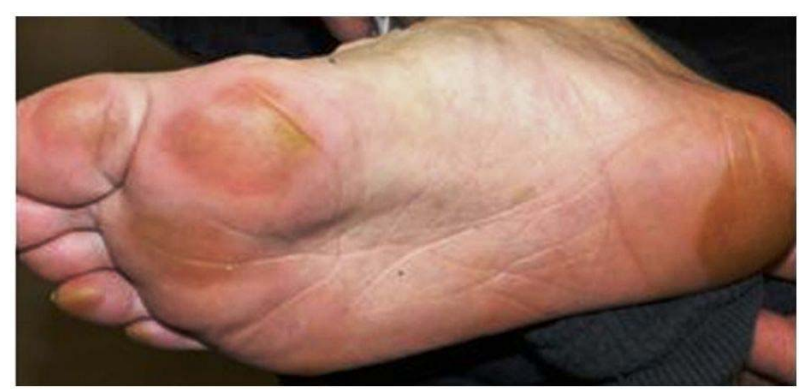

Figure 8. Hand-foot syndrome on foot.

mandibular molar region is believed to contribute to hematogeneous metastasis [15]. Hyposthesia of the lower lip is a crucial indication of metastasis [16]. In the present case, primary or metastatic mandibular tumor was suspected from the progressive hyposthesia of the lower lip. By several radiological examinations such as CT, scintigraphy and PET, the clinical diagnosis of renal tumor with multiple metastases was obtained. Biopsy of
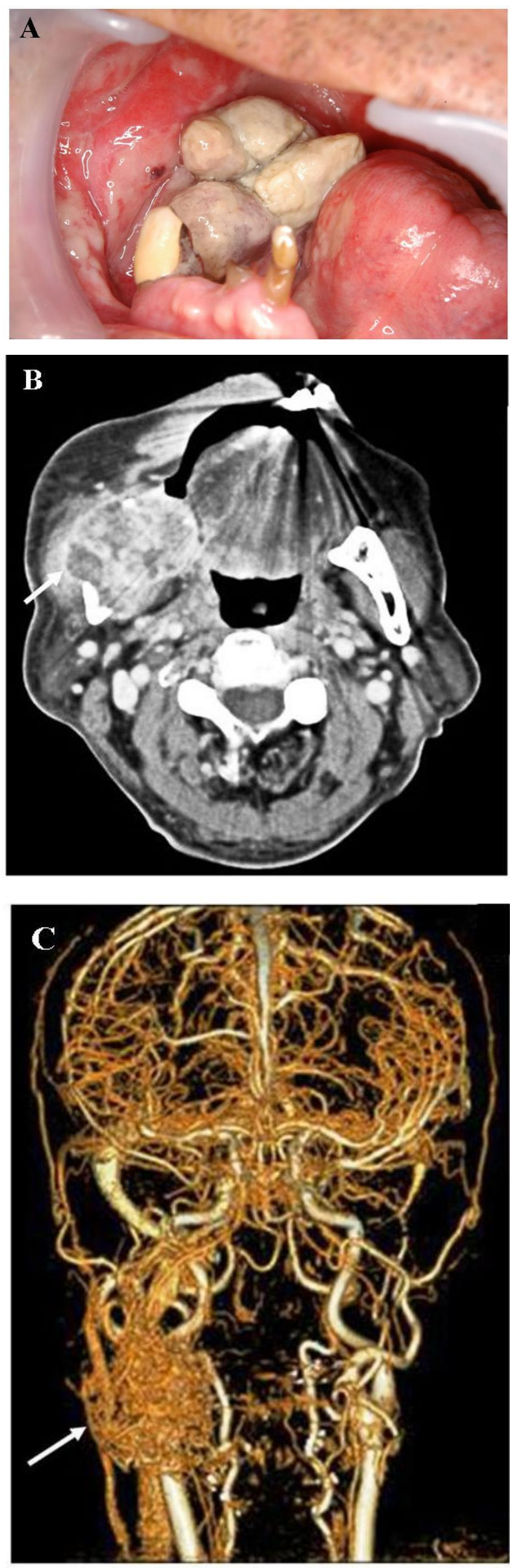

A: Oral finding: The mandibular metastatic lesion expanded markedly. The necrotic mass was exposed in the oral cavity; B: CT finding of the mandible: $51 \times 45 \mathrm{~mm}$ tumor was observed in the right mandibular body with destruction of buccal and lingual cortical bone (arrow); C: 3D angiography finding: The lesion was rich in blood vessels via infraalveolar and facial arteries (arrow).

Figure 9. Mandibular lesion 10 months after the start of molecular targeted therapy. 
the mandibular lesion was not performed for the definite diagnosis, since the diagnosis can be done during cytoreductive nephrectomy in selected patients with metastasis according to the National Comprehensive Cancer Network (NCCN) guideline [17].

The prognosis of patients with metastatic neoplasms in the oral region is poor $[10,11]$. Most patients die within 1 year since metastatic tumors in the oral region are often preceded or accompanied by multiple metastatic lesions at other sites and organs [9]. Sarcomatoid renal cell carcinoma, with metastasis at the initial diagnosis, shows an extremely poor prognosis with the median survival time of 6.3 months [4-6]. In the present case, 3 risk factors in predictors of short survival in NCCN guideline [17] (hemoglobin level below lower limit of normal, less than a year from original diagnosis to the start of systemic therapy, more than 2 sites of organ metastasis) were present. Therefore, the expected survival period was thought to be about 6 months in this case.

Mian [18] reported that the overall survival period was prolonged by nephrectomy and systemic therapy for sarcomatoid renal cell carcinoma. The NCCN guideline [17] also indicates radiation therapy for bone metastases in some instances for palliation of symptoms such as pain or cord compression. In the present case, radiation therapy was performed at first since endovascular aneurismal repair was necessary before any surgical treatment. Then cytoreductive radical nephrectomy and interferon therapy were performed. Surgery for the mandibular lesion was not indicated because extensive reconstruction is required after segmental resection including soft tissue due to the growth of the lesion.

Since interferon therapy was not effective in this case, a molecular targeted drug, Sorafenib, was administered. Sorafenib is an oral multikinase inhibitor that blocks tumour cell proliferation by targeting the Raf/MEK/ERK signalling pathway and exerts an antiangiogenic effect by targeting the tyrosine kinases of vascular endothelial growth factor receptor (VEGFR)-2, VEGFR-3, and platelet-derived growth factor receptor (PDGFR)-beta [18]. Sorafenib was reported to show a higher response rate in primary and metastatic sites of renal cell carcinoma with a favorable safety profile compared with cytokine-based immunotherapy and to prolong the progression-free survival (PFS) $[7,19,20]$. Sorafenib is also effective as a second-line drug with substantial anti-tumor activity for metastatic renal cell carcinoma after cytokine therapy [20]. The present patient tolerated Sorafenib therapy relatively well for a year. After hand-food syndrome developed at 1.5 months as a common adverse event [21], the therapy was continued with a lower dose, since rapid tumor revascularization and re-growth have been reported by the cessation of molecular targeted drugs [22].

Sorafenib effectively inhibited the growth of the me- tastatic lesions of sarcomatoid renal cell carcinoma for 10 months, and prolonged the survival period for as long as 2 years with a relatively good quality of life after the appearance of the first symptom; however, the metastatic lesions became resistant to the therapy and expanded after 10 months. Furthermore, severe adverse events, such as gastrointestinal perforation and hypothyroidism, also developed in the late stage [23]; therefore, molecular targeted medicines should be used with great care in combination with supportive therapies to alleviate the symptoms and to minimize severe adverse events.

In conclusion, a case of metastatic sarcomatoid renal cell carcinoma to the mandible is reported. Sorafenib effectively inhibited the growth of metastatic lesions for 10 months after conventional therapies and prolonged the survival period for as long as 2 years after the appearance of the first symptom. Molecular targeted therapy is thought to be a forefront choice with validity for such a case.

\section{ACKNOWLEDGEMENTS}

The authors would like to thank Kiyohide Fujimoto, MD, Associate Professor, Department of Urology, Nara Medical University, who performed the molecular targeted therapy to this patient.

\section{REFERENCES}

[1] Oscar, M.R., Raul, G.G., Jesus, M.A., et al. (2009) Metastasis of renal clear-cell carcinoma to the oral mucosa, an atypical location. Medicina Oral, Patologia Oral y Cirugia Bucal, 14, 601-604.

[2] Pritchyk, K.M., Schiff, B.A., Newkirk, K.A., et al. (2002) Metastatic renal carcinoma to the head and neck. The Laryngoscope, 112, 1598-1602. doi:10.1097/00005537-200209000-00012

[3] Montie, J.E., Stewart, B.H., Straffon, R.A., et al. (1977) The role of adjunctive nephrectomy in patients with metastatic renal cell carcinoma. The Journal of Urology, 117, 272-275.

[4] Tomera, K.M., Farrow, G.M. and Lieber, M.M. (1983) Sarcomatoid renal cell carcinoma. The Journal of Urology, 130, 657-659.

[5] Bertoni, F., Ferri, C., Benati, A., et al. (1987) Sarcomatoid renal cell carcinoma of kidney. The Journal of Urology, 137, 25-28.

[6] Ro, J.Y., Ayala, A.G., Sella, A., et al. (1987) Sarcomatoid renal cell carcinoma, clinicopathologic. A study of 42 cases. Cancer, 59, 516-526. doi:10.1002/1097-0142(19870201)59:3<516::AID-CNC R2820590327>3.0.CO;2-W

[7] Shuch, B., Riggs, S.B., LaRochelle, J.C., et al. (2008) Neoadjuvant targeted therapy and advanced kidney cancer: Observations and implications for a new treatment paradigm. BJU International, 102, 692-696. doi:10.1111/j.1464-410X.2008.07660.x

[8] Hirshberg, A., Leibovich, P. and Buchner, A. (1993) Me- 
tastasis to the oral mucosa: Analysis of 157 cases. Journal of Oral Pathology \& Medicine, 22, 385-390. doi:10.1111/j.1600-0714.1993.tb00128.x

[9] Fukuda, M., Miyata, M., Okabe, K., et al. (2002) A case series of 9 tumors metastatic to the oral and maxillofacial region. Journal of Oral and Maxillofacial Surgery, 60, 942-944. doi:10.1053/joms.2002.33868

[10] Meyer, I. and Shklar, G. (1965) Malignant tumors metastatic to mouth and jaws. Oral Surgery, Oral Medicine, Oral Pathology, 20, 350-362. doi:10.1016/0030-4220(65)90167-2

[11] Astacio, J.N. and Alfano, C. (1969) Oral mucosa metastasis from gastric adenocarcinoma. Oral Surgery, Oral Medicine, Oral Pathology, 28, 859-861. doi:10.1016/0030-4220(69)90339-9

[12] Nishmura, Y., Yakata, H., Kawasaki, T., et al. (1982) Metastatic tumors of the mouth and jaws: A review of the Japanese literature. Journal of Maxillofacial Surgery, 4, 253-258.

[13] Zachriades, N. (1980) Neoplasms metastatic to the mouth, jaw and surrounding tissues. Journal of Cranio-Maxillofacial Surgery, 17, 283-290. doi:10.1016/S1010-5182(89)80098-8

[14] Zohar, Y., Ben-Tovim, R., Gal, R., et al. (1985) Metastatic carcinoma of oral soft tissue. Head \& Neck Surgery, 7, 484-486. doi:10.1002/hed.2890070609

[15] Roser, S.M., Nicholas, T.R. and Hirose, F.M. (1976) Metastatic chondrosarcoma to the maxilla: Review of the literature and report of case. Journal of oral surgery, 34, 1012-1015.

[16] Glaser, C., Lang, S., Pruckmayer, M., et al. (1997) Clinical manifestations and diagnostic approach to metastatic cancer of the mandible. International Journal of Oral and Maxillofacial Surgery, 26, 365-368.
doi:10.1016/S0901-5027(97)80798-9

[17] NCCN Clinical Practice Guidelines in Oncology (2010) Kidney cancer.

http://www.tri-kobe.org/nccn/guideline/urological/english kidney.pdf

[18] Wilhelm, S.M., Carter, C., Tang, L., et al. (2004) Bay 43-9006 exhibits broad spectrum oral antitumor activity and targets the RAF/MEK/ERK pathway and receptor tyrosine kinases involved in tumor progression and angiogenesis. Cancer Research, 64, 7099-7109. doi:10.1158/0008-5472.CAN-04-1443

[19] Bukowski, R.M., Eisen, T., Szczylik, C., et al. (2007) Final results of the randomized phase trial of Sorafenib in advanced renal cell carcinoma: Survival and biomarker analysis. Journal of Clinical Oncology, 25, 5023.

[20] Motzer, R.J., Rini, B.I., Bukowski, R.M., et al. (2006) Activity of SU 11248, a multitargeted inhibitor of vascular endothelial growth factor receptor and platelet-derived growth factor receptor, in patients with metastatic renal cell carcinoma. Journal of Clinical Oncology, 24, 16-24. doi:10.1200/JCO.2005.02.2574

[21] Lacouture, M.E., Reilly, L.M., Gerami, P., et al. (2008) Hand foot skin reaction in cancer patients treated with the multikinase inhibitors sorafenib and sunitinib. Annals of Oncology, 19, 1955-1961. doi:10.1093/annonc/mdn389

[22] Nagengast, W.B., Lub-de Hooge, M.N., Oosting, S.F., et al. (2010) VEGF-PET imaging is a noninvasive biomarker showing differential changes in the tumor during sunitinib treatment. Cancer Research, 71, 143-153. doi:10.1158/0008-5472.CAN-10-1088

[23] Llovet, J.M., Ricci, S., Mazzaferro, V., et al. (2008) Sorafenib in advanced hepatocellular carcinoma. The New England Journal of Medicine, 359, 378-390. doi:10.1056/NEJMoa0708857 